\title{
Fetal alcohol spectrum disorder: reconsidering blame
}

\author{
Anna Maria Abadir BHSC MD, Abel Ickowicz MD MHSc
}

See also www.cmaj.ca/lookup/doi/10.1503/cmaj.141593

$\mathrm{F}$ etal alcohol spectrum disorder (FASD) is a widely accepted umbrella term used to encompass various effects of prenatal exposure to alcohol. The revised Canadian guideline for the diagnosis of FASD describes in detail the diagnostic requirements informed by evidence, such as sentinel facial features and impairment in specific neurodevelopmental capacities. ${ }^{1}$ Although the guideline emphasizes early detection and diagnosis of FASD as hallmarks for the provision of timely care and resources to those affected, far less attention is given to biological mothers. ${ }^{2}$ Here, we question and discuss common beliefs that have led to negative attitudes toward biological mothers of children with FASD.

The nefarious effects of prenatal alcohol exposure have been recognized for several hundred years; ${ }^{3}$ albeit, reference to alcohol teratogenicity appears much later in the health literature. ${ }^{4}$ Mothers of children with FASD are often viewed as having wilful problematic patterns of alcohol use, and being neglectful and unfit parents. Many people consider that drinking alcohol during pregnancy is a selfish act in which the mother gives more weight to her immediate inclinations than to the potential optimal health of her unborn child, either by intent or neglect. The erroneous belief that alcohol use disorder is a "disorder of will," and hence these mothers can and should make different choices, leads to an assumption that the FASD is entirely the mother's fault. However, ascribing blame to biological mothers of children with FASD is often unjustified, and careful reflection on emerging evidence is prudent.

The belief that pregnant women always drink alcohol deliberately while aware of their pregnancies and aware of the effects of alcohol on their progeny presupposes that they make a conscious decision to ignore information on harms and that fathers have no role to play. Population and qualitative studies ${ }^{2,5,6}$ have shown that many mothers of children with FASD do not have an alcohol use disorder. In many instances, mothers were unaware of the pregnancy at the time of alcohol consumption. A qualitative study in New Zealand that involved biological mothers living with their children affected by FASD, showed that many were ignorant of the effects of alcohol exposure on their developing offspring and had no knowledge of the potential risk of FASD while pregnant. ${ }^{2}$ Not surprisingly, in this study mothers at the highest risk of having children with FASD came from lower socioeconomic backgrounds, had lower education levels, had partners who used alcohol regularly, had inadequate nutrition during pregnancy, were undergoing stress or abuse during pregnancy and had reduced access to prenatal care. ${ }^{2}$

The teratogenic effects caused by alcohol exposure during pregnancy occur through complex gene-environment interactions during early development of the embryo, sometimes as early as gamete formation. ${ }^{7}$ Studies of alcohol exposure in rodents have identified important epigenetic effects in DNA methylation and histone modifications that result in changes in gene expression. It is important to note that the teratogenic potential of alcohol is present before conception and may affect would-be fathers as well as mothers. The impact of alcohol use by men during the preconception period and its consequences on progeny was recognized more than 100 years ago. ${ }^{7}$ Alcohol use by fathers before children are conceived has been associated with reduced birth weight and cognitive impairments. ${ }^{7}$ In the New Zealand study, many women stated that their husband or partner was drinking heavily at the time of conception. ${ }^{2}$

The revised FASD guideline appropriately identifies primary care physicians and front-line service providers as having a critical role in screening pregnant women for problematic alcohol and substance use. The guideline also stresses that

\section{KEY POINTS}

- Many mothers of children with fetal alcohol spectrum disorder (FASD) do not have problematic patterns of alcohol use and are not neglectful, unfit parents.

- The social determinants of health have a major role in the cause of FASD.

- Preconception consumption of alcohol by would-be fathers and mothers is linked to FASD.

- Preventive interventions should promote alcohol abstinence before conception as well as during pregnancy. 
abstinence should be recommended for all women during pregnancy to ensure the safest outcome for the fetus. ${ }^{1}$ In addition, we suggest that a strong emphasis should be placed on the avoidance of alcohol use before conception by men and women who are contemplating having children. Although it still plays an important role during prenatal care, education about the teratogenic risk associated with alcohol use needs to start much earlier. It is also necessary to take into consideration that the population at highest risk is characterized by marked psychosocial adversity; ${ }^{2}$ consequently, education alone falls short when the social determinants of health that impede patient access to care are ignored.

There is a clear benefit in taking active steps toward child protection when neglect or abuse is identified; however, it should be recognized that not all mothers of children with FASD are abusive or neglectful. Mothers of children with FASD often feel abandoned by the health care system and express much frustration at the attitudes of health care providers toward them. ${ }^{6}$ A blanketed attitude of blame and marginalization toward these mothers might indeed affect their sense of competency and ability to parent a child who already faces many challenges. Furthermore, blame becomes irrelevant when clinicians, policy-makers and the public collectively and actively assume responsibility for addressing the manifest determinants of health that contribute to the persistence of FASD.

There is no question that alcohol has a toxic effect on the unborn child. Our increasing understanding of the multifactorial nature of this phenomenon highlights the need to broaden our interventions, particularly in the realms of public health and prevention. We cannot globally attribute intentional neglect to all mothers of children with FASD. Assessments should be made on a case-by-case basis. Mothers and fathers who have children with FASD deserve to be treated with consideration and respect.

\section{References}

1. Cook JL, Green CR, Lilley CM, et al. Fetal alcohol spectrum disorder: a guideline for diagnosis across the lifespan. CMAJ 2015 Dec. 14 [Epub ahead of print].

2. Salmon J. Fetal alcohol spectrum disorder: New Zealand birth mothers' experiences. Can J Clin Pharmacol 2008;15:e191-213.

3. Abel EL. Gin Lane: Did Hogarth know about the fetal alcohol syndrome? Alcohol Alcohol 2001;36:131-4.

4. Jones KL, Smith DW. Recognition of the fetal alcohol syndrome in early infancy. Lancet 1973;302:999-1001.

5. Cannon MJ, Dominique Y, O'Leary LA, et al. Characteristics and behaviors of mothers who have a child with fetal alcohol syndrome. Neurotoxicol Teratol 2012;34:90-5.

6. Sanders JL, Buck G. A long journey: biological and non-biological parents' experiences raising children with FASD. J Popul Ther Clin Pharmacol 2010;17:e308-22.

7. Ramsay M. Genetic and epigenetic insights into fetal alcohol spectrum disorders. Genome Med 2010;2:27.

Affiliations: Department of Psychiatry (Abadir, Ickowicz), University of Toronto; Department of Psychiatry (Ickowicz), The Hospital for Sick Kids, Toronto, Ont.

Contributors: Both of the authors contributed equally to the conception and design of the manuscript, and the acquisition and interpretation of the available literature. Both of the authors drafted the manuscript, revised it critically for intellectual content, approved the final version to be published and agreed to act as guarantors of the work. 